Session 2259

\title{
Flowfield Mapping and Cooling Fan Flowrate Measurement Systems Development by Aerospace Engineering Laboratory Students
}

\author{
T. Hannigan, J. Simmons, K. Koenig, G. Cruse, K. Poh \\ Mississippi State University/USDA, Agricultural Research Service
}

Graduate and undergraduate students actively participated in a successful research project for a United States Department of Agriculture laboratory to monitor ventilation air flow in large poultry houses. Aerospace engineering laboratory students, graduate assistants, and faculty assisted in determining the flow rate through a stock cooling fan enclosure, evaluated and redesigned a prototype flow measurement device, and developed a large flowfield mapping system. Laboratory topics typically included data acquisition and control in air flows, including sensor selection, calibrations, and assessment of results, work on this research project could easily be substituted for normal laboratory experiences. Considerable design initiative, problem solving, program development for data acquisition, data reduction, error assessment, and uncertainty analysis were all required of students working on this project.

\section{Background}

Students take a two-course sequence of classes designed to bridge the gap between classroom and experimentation. The difficulties in validating analytical or numerical solutions to problems of aerodynamics are well established. Classical empirical and closed form solutions exist for many airflows of interest, but experimentation results that closely correlate with these solutions are not easily obtained. Lab basics include transducer design and selection, and methods of automating the data acquisition and control process. Such automation, coupled with data reduction and presentation on the personal computer, allows repeatable results by relatively inexperienced lab students. This repeatability minimizes incorrect correlation of errors associated with the method of data acquisition with those due to random errors in experimentation and analysis. Careful documentation and repetition of past successful experiments lead students to believe that there is no particular need for insight and experience in laboratory and field testing procedures.

In an effort to involve undergraduate students in research that was unique and open-ended, a cooperative research effort was established with a seemingly unrelated discipline, poultry science. Researchers from a USDA laboratory had contacted aerospace faculty, in an effort to obtain assistance in their experimentation and research into airflows ${ }^{1}$ in poultry houses. In the past decade, the expansion of the poultry industry has been great, and growth of meat-market birds has turned into a very large and diversified industry, revolving around growers on individual farms. Producers provide the growers with all necessary raw materials and then harvest and market their birds, once grown. Economic pressure is applied to the growers by producers, so grower attempts to more efficiently turn feed into marketable poundage have necessitated automation of the environment of the growing birds. Automatic feeding, watering, and ventilation systems have become a requirement in the industry. The most effective poultry 
houses have been those designed around a tunnel-house concept, where air is drawn into one end and exhausted from the other end of houses up to five hundred feet long.

Researchers ${ }^{2}$ have indicated that airflow patterns within tunnel houses is critical, particularly in hot weather. Fans are susceptible to wide variations in efficiency related to dampers, inlets, etc. However, there was no quick, reliable, repeatable test for fan performance, and total volume flow rate determination in the field. Procedures for standard field measurements ${ }^{3}$ were not considered adequate for specific research, and no equipment existed for mapping the very large flow field inside an actual tunnel house. The USDA lab offered funding for materials, travel and extra time spent on research beyond hours normally associated with the lab classes. In turn, the faculty, technicians, teaching assistants, and students all focused some time and attention on a current research problem--determining the flow rate of cooling fans in, and mapping the very large flow field of, an actual working tunnel house.

\section{Cooperative Research Effort}

Procedures were already established for cooperative research agreements between the Mississippi State University and the USDA Poultry Science Laboratory. In a series of informal meetings, objectives were established, and work on cooling fan research was subsequently integrated with and substituted for some laboratory class assignments.

Three related tasks were met in the development, calibration and field use of airflow measurement systems. The first task was to determine the flowrate through a cooling fan. Standard methods of determining the flowrate through a fan are detailed by the Air Movement and Control Association ${ }^{4}$. These methods require special equipment or test sections, are time consuming, and may require modification of the basic cooling fan installation. Alternative methods of assessing the flowrate through a given fan were sought, and a standoff flowrate measurement system was developed to allow quick and accurate positioning of a probe at various locations within a horizontally displaced flowfield. Positioning of the measurement probe was monitored on a computer screen as an arm was manipulated within the flowfield. Sample positions were marked on the screen for ready reference, and a detailed estimate of the flowrate was determined by numerical integration of the flowfield measurements. The method correlated very well with laboratory standard measurements of given fans. The apparatus developed for this task was immediately used by researchers in other projects. For this project, knowledge was combined from mechanics of materials, electronics, aerodynamics, systems design, computer programming, and other courses from the aerospace curricula.

The second task was the evaluation and refinement of a prototype device for field measurement of flowrates through cooling fans in tunnel ventilated poultry houses. The device consisted of a horizontal array of propeller anemometers that could be traversed vertically through a flow field, for determining an average flow rate. A concept demonstrator was developed into an actual preproduction prototype design, which was constructed in the laboratory. This required digital and analog circuitry design, material selection and specification, enclosure and movement mechanism design, program development, and critical evaluation and calibration prior to field use in poultry research. This prototype was calibrated in aerospace laboratory tests, and in a fan manufacturers 
standard calibration laboratory, where the refined prototype compared very favorably against standard measurement methods. A standoff data acquisition and control system was developed using a personal computer, and the device was field tested successfully in an actual operating tunnel house. Even the original concept demonstrator was modified and adopted for use in a related research project.

The third related task was development of a system for mapping of large-scale flowfields. A vertical array of propeller anemometers, a horizontal positioning system, and a computer data acquisition and analysis method were developed for 3D mapping of flow in a 40 feet wide by 500 feet long tunnel house. Results obtained from research into factors impacting on the quality and quantity of flow in tunnel ventilated houses were immediately utilized in economic decisions by poultry growers. This project encouraged student involvement in innovative, cooperative, diversified, instrumentation analysis and design at an applied research level. These activities achieved course objectives better than canned exercises normally accomplished in lab classes.

\section{Student Initiative \& Development}

Given the initial task of determining the flow rate through a given fan, the students tried various methods that were typically used in calibrating and surveying flow fields in wind tunnels and related experiments. AMCA standard field methods detailed for measuring flow in cooling fan installations were not utilized, since an initial criteria was the development of an alternative method.

In a classic laboratory experiment normally conducted, a pitot static probe is traversed through a pipe flow well downstream from an inlet duct, to get a flow profile that can be integrated to determine flow rate. At the same time, flow volume is captured in a large plastic envelope that expands to full in a reasonably long period of time. A similar method was employed by the students to try to determine the normal output of a cooling fan. A continuous roll of semi-rigid plastic sheeting was obtained from a laundry supply, and two 100 foot long tubes were fashioned to collect flow from a fan. The two tubes were mated to a frame that could be swung into position on the outlet end of a fan. The

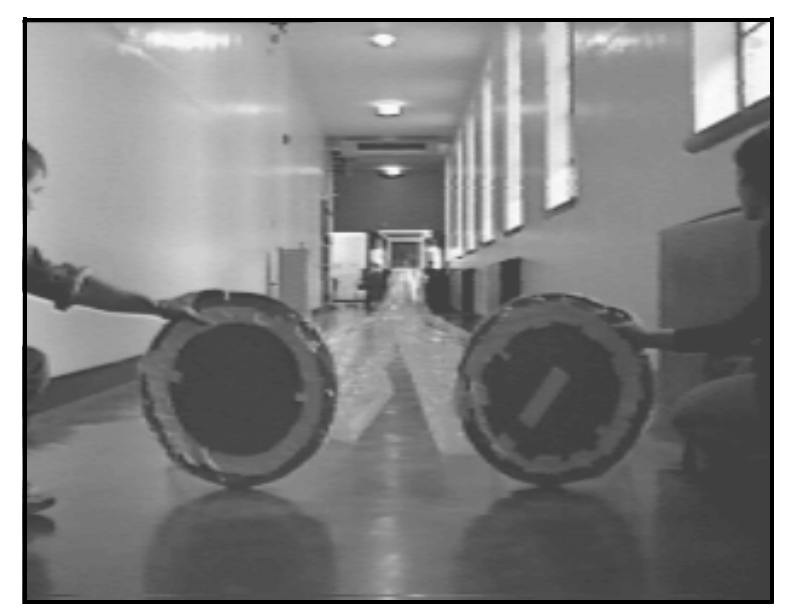

Figure 1 Total flow containment tubes with popout ends tubes would "pop" to their fully inflated form, and a "popout" plug would release when the tubes were full. A video camera was set up to film the inflation sequence, and the $30 \mathrm{~Hz}$ framing of the video was used to time the interval required to fill the volume.

The students were able to get consistent readings that were slightly less than the manufacturer's rating for the fan. The voltage and current requirements for the fans were measured, and it was 
determined that while the power required to run the fans remained near constant during the tests, a check of the fan rpm with a strobetac revealed that the rpm decreased slightly when the tubes were in place, resulting in the flowrate of the fans measuring less than expected due to flow restriction.

An eight foot long, four feet square extension was constructed downstream from the fan, with the intention of measuring flow with a pitot tube array at the exit of the extension. The extension was deliberately larger than the fan, and was in fact as large as the box typically used to mount this type cooling fan in a wall. In lieu of an array, a single probe was mounted at the end of an arm mounted on a telescoping stand that could be

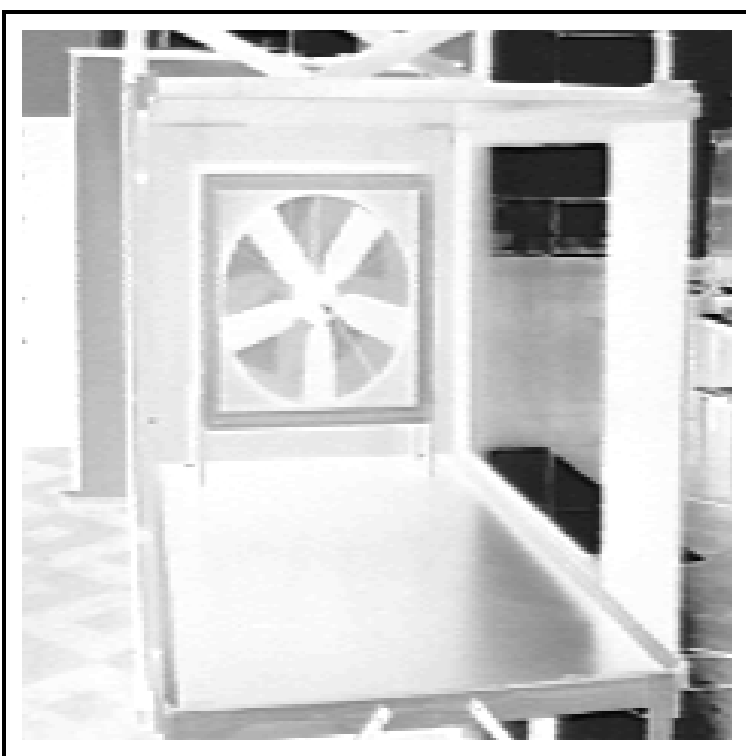

Figure 2

Fan box with extension attached positioned to one side, in the exit plane of the fan extension. The arm could be pivoted up and down, and extended back and forth in that plane. On the opposite side of the pivot, two rigid arms fastened to potentiometers allowed the accurate determination of the position of the probe, with simple geometric relations determined from the angles of the two arms of fixed lengths. Corrections to the probe position due to the weight of the probe causing flexure was a matter of applying Castigliano's Theorem. Tests of the probe position indicated that the probe could be placed within a four by four foot flow field plane with .25 -inch accuracy. Computer programs normally developed for other lab experiments were adapted specifically for this device, to collect voltages from the pots and the pitot probe transducer.

A program was developed that showed the desired sampling positions of the probe, and marked positions during the sampling, so that the arm could be manipulated within the flowfield by monitoring the computer screen. Samples were taken when a remote switch was pressed at the handle used to position the probe. Once a number of samples had been taken at intervals throughout the flow field, a process that took only a few minutes, this data could be used to perform a numerical integration of the flow field, thus establishing the flow rate. A program treating four adjacent samples as corners of a general quadrilateral were averaged as the average flow over the area, and the product of the area and flow summed over the flow field. The volume flow rate thus obtained was divided by the area to establish an average flow velocity. Precision of

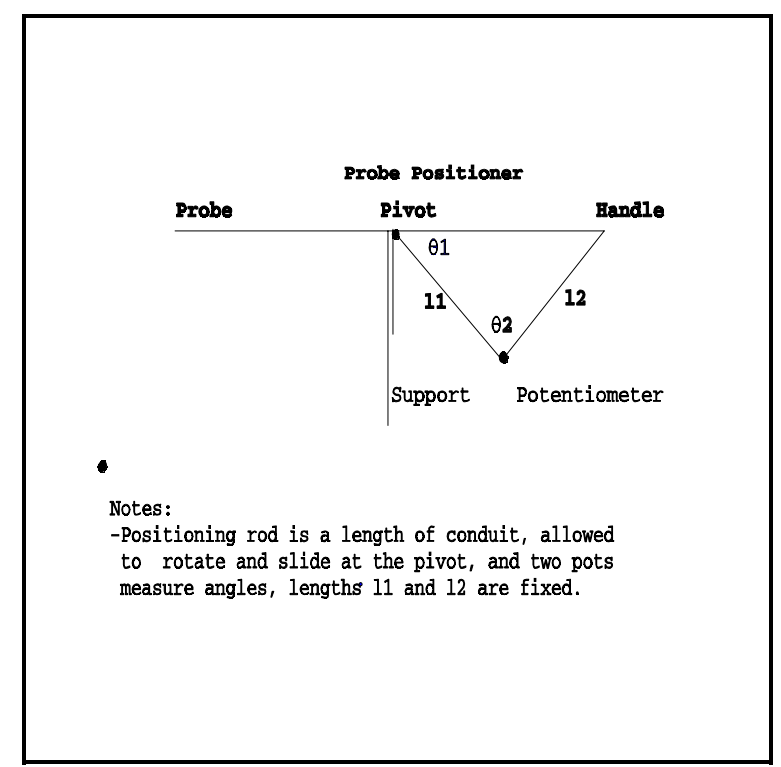

Figure 3 Probe positioning arm 
results was limited by the pressure transducer, so a propeller anemometer was used, which gave excellent low-speed results. This device was used in other student experiments to measure the flow field behind a propeller in the large subsonic wind tunnel, and downstream of an aircraft propeller, as well as giving a good survey of the flow field within the lab's subsonic wind tunnel.

The prototype flow measurement device provided by the USDA researchers was evaluated by coupling it to the inlet side of the fan, and concurrent measurements were obtained upstream and dowstream of the fan. A fog generator was used to study the flow through the inlet of the measurement system, fan, and tunnel. It was determined that there should be a fairing at the entrance to insure smooth flow into the fan. This in turn showed more stable flow patterns downstream of the fan, instead of a core flow that would rotate and move about the extension.

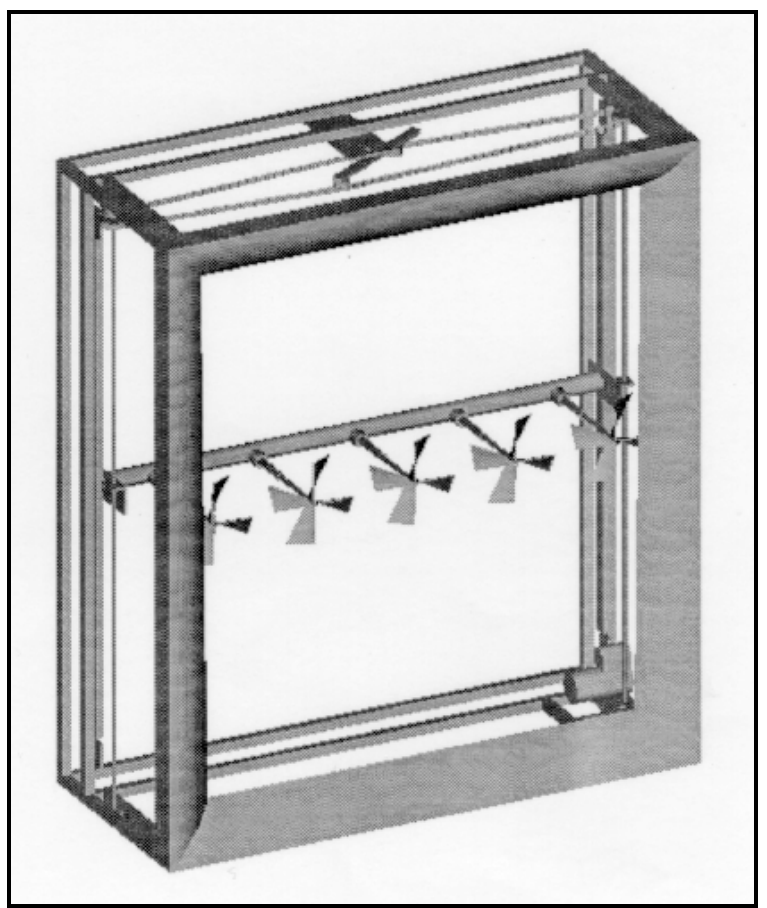

Figure 4 Propeller Anemometer Array (outer skin removed)

Experimentation quickly showed that a smooth fairing into the entrance of the measurement device allowed consistent measurements of the average flow rate through the inlet, and these results correlated well with downstream values. Based on the success of these tests, an improved prototype was designed primarily by the students, and was constructed by the lab technician with student assistance. The improved device was very easy to position, and gave very consistent results. Meanwhile, the prototype flow measurement device was pressed into service monitoring a research tunnel where live birds were being subjected to various flow conditions.

\section{Beyond the Laboratory}

To accomplish the measurement of the very large flow field in an actual poultry house, a vertical array of propeller anemometers free to pivot in a horizontal plane was constructed. The device was positioned at various intervals along, and traversed across the house, supported by cables strung top and bottom. A computer was used for data acquisition via an 85' umbilical, thus eliminating mechanical interference from experimenters in the vicinity of the measurement array during sampling. Maps of the flow field illustrated problems associated with deflector currents hung at intervals down the house, and areas of substantially reduced flow were obvious. These correlated with areas within the house where birds suffered respiratory and heat stress. Solutions to provide a more uniform flow field became obvious, as configuration changes were made, and the flow field was resampled.

The USDA fan inlet measurement device was used to determine the effect of such factors as dirty entrance fairings and louvres, exit extensions, and the lowering of the deflector curtains, or baffles. Flow fields were made visible within the house as a fog generator used to illustrate 
precise entrance flow field behavior, dead regions, and to determine the actual time required for air to make it from entrance to exit of the house. Recirculation patterns between baffles were also apparent, as were the effects of mechanical interference of equipment, heaters, feeders, etc.

Finally, in an effort to lend more plausibility to the fundamentally new method of determining fan flowrates in field testing, a trip was made to a standard laboratory at a fan manufacturer. The fans used in lab calibrations were re-tested to insure that they performed as rated, and simultaneous results were obtained with the USDA measurement device. The results correlated very well, and the validity of the device was established. USDA researchers are actively using the device in further tests of adjacent fan interference, and for research into factors influencing fan position and selection.

\section{Conclusions}

This was an extremely exciting and successful endeavor. The students enjoyed various experiments, particularly the flow visualization efforts, and the on-the-fly development of problem solutions, such as the entrance fairings. Brain-storming sessions were held at various intervals during this process, and many small and individually unique ideas were brought together as a whole to solve a problem at hand. Students learned to work together, to improvise, and to actually use what they had learned, from electronics to aerodynamics, from structures to programming, and they had a lot of fun along the way. Visits to a modern poultry farm surprised the students, particularly in that a high degree of automation had found its way to such endeavors.

They saw that process control learned in the classroom had fundamental economic impact on the efficiency of a real phenomena--the growth of chickens. They saw the development of a prototype device through product refinement, including cost determination, market potential, and patent consideration. This project lasted through three semesters, with all of the lab testing and development being done for lab credit, and field testing being done by paid student workers and graduate assistants during the summer. Only a few of the normal classic experiments were displaced by these projects, but student interest and ability to adapt seemed markedly higher than in past years, particularly in experiments done in lab after working on this project. The students were not satisfied to just do an experiment in the manner it was presented. They wanted to modify and improve the experimental methods. In short, they wanted to practice engineering.

\section{Future Plans}

Interest in having students work on research projects is obviously on the upswing. This past semester, the students were solicited to instrument and then measure vibration amplitudes and frequencies for a forest products process apparatus, which they successfully did in lieu of an experiment normally done on a simple cantilever beam. They have also performed wind tunnel dynamic and static testing and calibrations of air data probes for an independent engineering

firm. A new and continuing cooperative research agreement is in the works for the poultry science lab. 
1. Hannigan, T., Koenig, T. Cruse, G., Poh, K., "Calibration and Testing of Anemometry Equipment for a Cooling Fan Volume Flow Rate Measurement System" MSSU-EIRS-ASE-94-4, MSU, MS, June 1995.

2. Czarick, M., Lacy, M., "Uniformity of air movement is key to tunnel-ventilated houses" Poultry Times, 11 March 1996.

3. Field Performance Measurement of Fan Systems, AMCA Publication 203-90, Arlington Heights, IL, 1990.

4. Laboratory Methods of Testing Fans for Rating, ANSI/AMCA Standard 210-85, Arlington Heights, IL, 1986.

THOMAS HANNIGAN is the coordinator for laboratories and informations systems administrator of the Aerospace Engineering Department, Mississippi State University. He is an instructor in the Aerospace Engineering Department, and focuses on development of laboratory experiences for undergraduates, outreach programs to K-12, aviation laboratory applications, and non-traditional aerospace applications. M.S. Aerospace Engineering, Mississippi State University. Flight Instructor, Airplane Single \& Multi-Engine and Instrument.

JOHN SIMMONS is a research engineer for the United States Department of Agriculture, Agricultural Research Service, South Central Poultry Research Laboratory. He currently specializes in research concerning ventilation and cooling optimization in poultry houses. Ph.D., Clemson University.

KEITH KOENIG is a professor of Aerospace Engineering at Mississippi State University, focusing on fluid mechanics, aerodynamics, structural dynamics and propulsion. He is also active in outreach activities to K-12, and non-traditional applications of aerospace research, such as sports applications. Ph.D., Aeronautics, California Institute of Technology.

GREG CRUSE served as engineering technician in the senior aerospace engineering laboratories. He assisted students with individual seminar project development, and with the conduct of general laboratory experiments and research. B.S., Electrical Engineering Technology, University of Southern Mississippi, M.S., Aerospace Engineering, Mississippi State University.

KOKHONG POH served as graduate teaching assistant in the senior aerospace engineering laboratories while working on his M.S. He assisted and monitored student participation in individual and collective research. M.S., Aerospace Engineering, Mississippi State University. 